\title{
On the spectrum of the Schrödinger operator on $\mathbb{T}^{d}$ : a normal form approach
}

Dario Bambusi* Beatrice Langellał, Riccardo Montalto $\ddagger$

\begin{abstract}
In this paper we study the spectrum of the operator

$$
H:=(-\Delta)^{M / 2}+\mathcal{V}, \quad M>0,
$$

on $L^{2}\left(\mathbb{R}^{d} / \Gamma\right)$, with $\Gamma$ a maximal dimension lattice in $\mathbb{R}^{d}$ and $\mathcal{V}$ a pseudodifferential operator of order strictly smaller than $M$. We prove that most of its eigenvalues admit the asymptotic expansion

$$
\lambda_{\xi}=|\xi|^{M}+Z(\xi)+O\left(|\xi|^{-\infty}\right),
$$

where $Z$ is a $C^{\infty}\left(\mathbb{R}^{d}\right)$ function (symbol) and $\xi \in \Gamma^{*}$ (the dual lattice
\end{abstract} of $\Gamma)$.

Keywords: Schrödinger operator, normal form, pseudo differential operators

MSC 2010: 37K10, 35Q55

\section{Introduction}

Let $\Gamma$ be a lattice of dimension $d$ in $\mathbb{R}^{d}$, with basis $\mathbf{e}_{1}, \mathbf{e}_{2}, \ldots, \mathbf{e}_{d}$, namely

$$
\Gamma:=\left\{\sum_{i=1}^{d} k_{i} \mathbf{e}_{i}: k_{1}, \ldots, k_{d} \in \mathbb{Z}\right\},
$$

${ }^{*}$ Dipartimento di Matematica, Università degli Studi di Milano, Via Saldini 50, I-20133 Milano. Email: dario.bambusi@unimi.it

${ }^{\dagger}$ Dipartimento di Matematica, Università degli Studi di Milano, Via Saldini 50, I-20133 Milano. Email: beatrice.langella@unimi.it

${ }^{\ddagger}$ Dipartimento di Matematica, Università degli Studi di Milano, Via Saldini 50, I-20133 Milano. Email: riccardo.montalto@unimi.it 
and define

$$
\mathbb{T}_{\Gamma}^{d}:=\mathbb{R}^{d} / \Gamma
$$

In this paper we study the asymptotic behavior of a large part of the spectrum of the operator $(0.1)$ in $L^{2}\left(\mathbb{T}_{\Gamma}^{d}\right)$ and we prove that there exists a smooth function (actually a symbol admitting a full asymptotic expansion) $Z(\xi)$ s.t. for most points $\xi \in \Gamma^{*}$ the corresponding eigenvalue $\lambda_{\xi}$ is given by $(0.2)$. By most points we intend that they form a set of density one (for a more precise estimate see eq. (2.11) below). We remark that a particular case which is included in our theory is that of the classical Sturm Liouville operators

$$
-\Delta+V(x)
$$

with periodic or Floquet boundary conditions.

The spectrum of the Sturm Liouville operator (1.3) was studied in [W] and then in FKT90, Fri90, whose results are in the same spirit of our ones. We recall that in [FKT90] it was proven that if $V$ is a sufficiently smooth potential with zero average and the lattice is generic, then, for $\xi$ in a set of density 1 , there are two eigenvalues $\lambda_{ \pm \xi}$ in the interval

$$
\left[|\xi|^{2}-\frac{1}{|\xi|^{2-\epsilon}},|\xi|^{2}+\frac{1}{|\xi|^{2-\epsilon}}\right] \text {. }
$$

In [FKT90] the result was proven for $d=2,3$, while in [Fri90 the result was extended to general dimension (for further results see [Vel15, Wan11]).

Karpeshina [Kar96, Kar97] studied in detail the case of Floquet boundary conditions for bounded perturbations. She proved that, generically (in the Floquet parameters), most eigenvalues are simple and she gave a full asymptotic expansion of each one of these simple eigenvalues.

In the present paper we get a full asymptotic expansion of the eigenvalues studied in [FKT90, Fri90], getting in particular that, for $C^{\infty}$ potentials, one has

$$
\lambda_{\xi}-\lambda_{-\xi}=O\left(|\xi|^{-\infty}\right) .
$$

Such a property is well known in dimension 1, but, as far as we know, was not known in higher dimensions. Furthermore the main improvement that we get here is that we are able to deal with unbounded perturbations.

The proof is a development of the procedure already used in Bam18, Bam17, BM18 (see also BBM14, BGMR18) and consists of a normal form procedure allowing to conjugate the operator (0.1) to a Fourier multiplier plus an operator which is smoothing at all orders. This is constructed by quantizing the classical normal form procedure applied to the symbol of the operator $H$. The main difference with respect to Bam18, Bam17, BM18] 
is that in our problem the resonances of the classical Hamiltonian system corresponding to the unperturbed operator $(-\Delta$, in our case) depend on the point of the phase space, so we restrict our construction to the nonresonant regions of the phase space. This is the reason why we only get the result for most eigenvalues. A detailed heuristic description of the proof is given in Sect. 3. We recall that a similar procedure was also developed in a semiclassical context in Roy07.

A question that we do not address here is that of the behavior of the part of the spectrum corresponding to the resonant zones of the phase space. This will be the object of a separate study.

We remark that a normal form theorem with some similarities with the one presented here was obtained in [PS10] (see Theorem 4.3). However, as far as we know, our results on the periodic eigenvalues of the operator (0.1) are new. Furthermore we think that our proof of the normal form theorem, which is based on symbolic calculus, is simpler than that of [PS10] and could also have some interest.

The paper is organized as follows: in Section 2 we state our main result, see Theorem 2.4. In Section 3 we explain roughly the strategy of our proof. In Section 4 we recall some standard facts on the theory of pseudo-differential operators. In Section 5 we state and prove our normal form result (see Theorem 5.1) and in Section 6 we show how Theorem 2.4 can be deduced from it.

Acknowledgments: This research is supported by GNFM. We warmly thank Thomas Kappeler for suggesting some references on the topic and Emanuele Haus, Alberto Maspero and Michela Procesi for many useful discussions and comments.

\section{Main result}

First we recall that the dual lattice $\Gamma^{*}$ is defined by

$$
\Gamma^{*}:=\left\{b \in \mathbb{R}^{d}: b \cdot k \in 2 \pi \mathbb{Z}, \quad \forall k \in \Gamma\right\}
$$

Given any function $u \in L^{2}\left(\mathbb{T}_{\Gamma}^{d}\right)$, it can be expanded in Fourier series as

$$
u(x)=\sum_{\xi \in \Gamma^{*}} \widehat{u}(\xi) e^{\mathrm{i} x \cdot \xi}, \quad \widehat{u}(\xi):=\frac{1}{\left|\mathbb{T}_{\Gamma}^{d}\right|} \int_{\mathbb{T}_{\Gamma}^{d}} u(x) e^{-\mathrm{i} \xi \cdot x} d x, \quad \xi \in \Gamma^{*},
$$

where we denoted by $\left|\mathbb{T}_{\Gamma}^{d}\right|$ the Lebesgue measure of $\left|\mathbb{T}_{\Gamma}^{d}\right|$. More generally we will denote by $|\mathcal{A}|$ the measure of a measurable set $\mathcal{A}$. For any $s \geq 0$, we 
also introduce the Sobolev space $H^{s}\left(\mathbb{T}_{\Gamma}^{d}\right)$ defined by

$$
H^{s}\left(\mathbb{T}_{\Gamma}^{d}\right):=\left\{u \in L^{2}\left(\mathbb{T}_{\Gamma}^{d}\right):\|u\|_{H^{s}}:=\left(\sum_{\xi \in \Gamma^{*}}\langle\xi\rangle^{2 s}|\widehat{u}(\xi)|^{2}\right)^{\frac{1}{2}}<\infty\right\}
$$

where for any $\xi \in \mathbb{R}^{d}$, we set $\langle\xi\rangle:=\left(1+|\xi|^{2}\right)^{\frac{1}{2}}$.

Definition 2.1. Given $\delta>0$ and $m \in \mathbb{R}$ we define the symbol class $S_{\delta}^{m}$ as the set of all the functions $a \in C^{\infty}\left(\mathbb{T}_{\Gamma}^{d} \times \mathbb{R}^{d}\right)$ such that for any $\alpha, \beta \in \mathbb{N}^{d}$, there exists $C_{\alpha, \beta}$ s.t.

$$
\left|\partial_{x}^{\alpha} \partial_{\xi}^{\beta} a(x, \xi)\right| \leq C_{\alpha, \beta}\langle\xi\rangle^{m-\delta|\beta|}, \quad \forall(x, \xi) \in \mathbb{T}_{\Gamma}^{d} \times \mathbb{R}^{d} .
$$

Given a symbol $a \in S_{\delta}^{m}$, we define its Fourier coefficients w.r. to the variable $x$ as

$$
\widehat{a}(k, \xi):=\frac{1}{\left|\mathbb{T}_{\Gamma}^{d}\right|} \int_{\mathbb{T}_{\Gamma}^{d}} a(x, \xi) e^{-\mathrm{i} k \cdot x} d x, \quad \forall(k, \xi) \in \Gamma^{*} \times \mathbb{R}^{d},
$$

and the Weyl quantization of a symbol $a \in S_{\delta}^{m}$.

Definition 2.2 (Weyl quantization). Given a symbol $a \in S_{\delta}^{m}$, we define its Weyl quantization $O p^{w}(a)$ as follows: given $u \in C^{\infty}\left(\mathbb{T}_{\Gamma}^{d}\right)$, we put

$$
\left[O p^{w}(a) u\right](x)=\sum_{k, \xi \in \Gamma^{*}} \widehat{a}\left(k-\xi, \frac{k+\xi}{2}\right) \widehat{u}(\xi) e^{\mathrm{i} k \cdot x} .
$$

Correspondingly, we will say that an operator $A$ is pseudodifferential of class $O P S_{\delta}^{m}$ if there exists a symbol $a \in S_{\delta}^{m}$ such that $A=O p^{w}(a)$

Definition 2.3. Given a sequence of symbols $\left\{f_{j}\right\}_{j \geq 0}$ with $f_{j} \in S_{\delta}^{m-\rho j}$ for some $m \in \mathbb{R}$ and $\rho>0$, and a function $f(x, \xi)$, possibly defined only on $\mathbb{T}_{\Gamma} \times \Gamma^{*}$, we write

$$
f \sim \sum_{j} f_{j}
$$

if for any $N$ there exists $C_{N}$ s.t.

$$
\left|f(x, \xi)-\sum_{j=0}^{N} f_{j}(x, \xi)\right| \leq C_{N}\langle\xi\rangle^{m-(N+1) \rho} .
$$

If $f$ is defined only on $\mathbb{T}_{\Gamma} \times \Gamma^{*}$ then eq. 2.7) is valid in such a set. 
The main object of the paper is the spectrum of the operator 0.1 . We assume that there exists $0<\delta<1$ and $\mathfrak{e}>0$ s.t.

$$
\mathcal{V} \in O P S_{\delta}^{M-\mathfrak{e}}, \quad \text { and } \quad 1-\frac{\mathfrak{e}}{4}<\delta<1
$$

and that $\mathcal{V}$ is selfadjoint.

Define

$$
\rho:=4 \delta+\mathfrak{e}-4>0 .
$$

Note that the condition $(2.8)$ on $\delta$ implies that $\rho>0$. Denote by $B_{R}(x)$ the open ball of $\mathbb{R}^{d}$ having radius $R$ and center $x, B_{R}:=B_{R}(0)$ and denote by $\sharp E$ the counting measure of a set $E$.

Our main result is the following theorem.

Theorem 2.4. Consider the operator

$$
H:=[-\Delta]^{M / 2}+\mathcal{V},
$$

with $\mathcal{V}$ fulfilling 2.8 . There exists a set $\Omega \subset \mathbb{R}^{d}$, such that $\Omega \cap \Gamma^{*}$ has density one, more precisely one has

$$
1-\frac{\sharp\left(\Omega \cap \Gamma^{*} \cap B_{R}\right)}{\sharp\left(B_{R} \cap \Gamma^{*}\right)}=\mathcal{O}\left(R^{\delta-1}\right)
$$

and a sequence of symbols $z_{j} \in S^{M-\mathfrak{e}-j \rho}$, which depend on $\xi$ only, with the following property: for any $\xi \in \Omega \cap \Gamma^{*}$ there exists an eigenvalue $\lambda_{\xi}$ of (2.10) which admits the asymptotic expansion

$$
\lambda_{\xi} \sim|\xi|^{M}+\sum_{j \geq 0} z_{j}(\xi), \quad \xi \in \Omega \cap \Gamma^{*} .
$$

Furthermore, if the symbol $v(x, \xi)$ of $\mathcal{V}$ is symmetric with respect to $\xi$, namely $v(x, \xi)=v(x,-\xi)$, then $\xi \in \Omega$ implies $-\xi \in \Omega$ and one also has $z_{j}(\xi)=$ $z_{j}(-\xi), \forall j$.

Remark 2.5. Actually corresponding to any $\xi \in \Omega \cap \Gamma^{*}$ we construct a quasimode $\varphi_{\xi}$ with the property that for $\xi \neq \xi^{\prime}$ one has $\left\langle\varphi_{\xi}, \varphi_{\xi^{\prime}}\right\rangle_{L^{2}}=0$, so we construct a number of eigenvalues in one to one correspondence with $\Omega \cap \Gamma^{*}$, even in the case of multiple eigenvalues, which have to be counted with multiplicity.

Remark 2.6. Theorem 2.4 applies also to the case of Floquet boundary conditions:

$$
u(x+\gamma)=e^{\mathrm{i} \gamma \cdot \kappa} u(x), \quad \gamma \in \Gamma .
$$

Indeed, the operator (0.1) with Floquet boundary conditions is unitary equivalent to the operator with symbol $|\xi-\kappa|^{M}+v(x, \xi-\kappa)$, which of course fulfills the assumptions of Theorem 2.4. 
Remark 2.7. In the symmetric case, which in particular is true for the operator $-\Delta+V(x)$, one has that, for all $\xi \in \Omega$,

$$
\lambda_{\xi}-\lambda_{-\xi}=O\left(|\xi|^{-\infty}\right) \text {. }
$$

\section{Scheme of the proof}

The idea of the proof is to perform a "semiclassical normal form" (see e.g. [Bam04]) working on the symbol of $H$, namely to quantize the classical normal form procedure for the symbol of $H$.

To explain the algorithm we consider the simple case in which

$$
H=-\Delta+V(x)
$$

and $\Gamma=\mathbb{Z}^{d}$, namely the lattice generated by the canonical basis of $\mathbb{R}^{d}$. In this case $H$ is the Weyl quantization of the classical Hamiltonian

$$
h:=|\xi|^{2}+V(x) .
$$

We are interested in studying the system in the region $\langle\xi\rangle \gg 1$, in which the potential is a perturbation of the term $|\xi|^{2}$. Taking this point of view the perturbative parameter is $\langle\xi\rangle^{-1}$. Remark also that this corresponds to an expansion in lower order pseudodifferential operators after quantization.

The classical normal form procedure consists of looking for an auxiliary Hamiltonian function $g$ s.t. the corresponding time 1 flow $\phi_{g}^{1}$ (namely the time one flow of the corresponding Hamiltonian system), conjugates $h$ to a new Hamiltonian $h \circ \phi_{g}^{1}$ in which the dependence on the angles $x$ is pushed to higher order. It is well known that this can be done only in the nonresonant regions of the action space. The definition of the resonant regions is a key step of our procedure, hence we are now going to describe it.

By a formal computation one has

$$
h \circ \phi_{g}=h+\left\{|\xi|^{2} ; g\right\}+V+\text { lower order terms },
$$

where $\{. ;$.$\} is the Poisson bracket. The idea is to determine g$ in such a way that

$$
\left\{|\xi|^{2} ; g\right\}+V=\text { function of } \xi \text { only . }
$$

Expanding $g$ and $V$ in Fourier series in $x$, equation 3.2 takes the form

$$
\mathrm{i}(k \cdot \xi) \hat{g}(k, \xi)=\hat{V}(k, \xi) \Longleftrightarrow \hat{g}(k, \xi)=\frac{\hat{V}(k)}{\mathrm{i}(k \cdot \xi)}, \quad \forall k \in \mathbb{Z}^{d} \backslash\{0\}, \quad \xi \in \mathbb{R}^{d}
$$


so that the corresponding function $g$ would turn out to be singular at the dense subset

$$
\bigcup_{k \in \mathbb{Z}^{d} \backslash\{0\}}\left\{\xi \in \mathbb{R}^{d}: \xi \cdot k=0\right\} .
$$

In classical mechanics it is well known how to solve this problem: first take advantage of the decay of $|\hat{V}(k)|$ with $|k|$ in order to restrict the union to a finite subset of $\mathbb{Z}^{d}$, and then remove from the phase space a neighborhood of the so obtained set.

Since in our case the small parameter is $\langle\xi\rangle^{-1}$, and we are in a $C^{\infty}$ context, so that $|\hat{V}(k, \xi)|$ decays faster then any inverse power of $|k|$, we can proceed as follows: we fix some $\epsilon>0$ and define

$$
g(x, \xi):=\sum_{0<|k|<\langle\xi\rangle^{\epsilon}} \frac{\hat{V}(k, \xi)}{\mathrm{i} k \cdot \xi} \mathrm{e}^{i k \cdot x},
$$

but only on the set

$$
\left\{\xi \in \mathbb{R}^{d}:|\xi \cdot k|>\frac{\mu}{|k|^{\tau}}, \quad \forall k \in \mathbb{Z}^{d}, \quad 0<|k|<\langle\xi\rangle^{\epsilon},\right\} .
$$

However, even if $g$ is well defined and smooth on the set (3.5), this choice still has a problem: $g$ does not decay as $\langle\xi\rangle \rightarrow \infty$, since the $k$-th term of the sum (3.4) decays only in the direction $k$. The last remark for the classical case is that in the domain $|k \cdot \xi| \geq C\langle\xi\rangle^{\delta}$, with some $\delta>0$, the $k$-th term at r.h.s. of $(3.4)$ decays as $\langle\xi\rangle^{-\delta}$. This leads to the choice

$$
\mu=\langle\xi\rangle^{\delta}
$$

in the formula (3.5). So, we use $g$, but restricted to the domain

$$
\Omega^{(0)}:=\left\{\xi \in \mathbb{R}^{d}:|\xi \cdot k|>\frac{\langle\xi\rangle^{\delta}}{|k|^{\tau}}, \quad \forall k \in \mathbb{Z}^{d}, \quad 0<|k|<\langle\xi\rangle^{\epsilon}\right\} .
$$

Furthermore, the measure of the set $\Omega^{(0)}$ is asymptotically full in the sense that

$$
1-\frac{\left|\Omega^{(0)} \cap B_{R}\right|}{\left|B_{R}\right|}=O\left(R^{\delta-1}\right) .
$$

This is the classical procedure that we quantize. As usual in semiclassical normal form theory, the main remark is that, if $g \in S_{\delta}^{m}$ is a real valued symbol with $m<\delta$ and $G=O p^{w}(g)$, then $\mathrm{e}^{-\mathrm{i} G}$ is unitary, the operator $\mathrm{e}^{\mathrm{i} G} H \mathrm{e}^{-\mathrm{i} G}$ is pseudodifferential and is given by

$$
\mathrm{e}^{\mathrm{i} G} H \mathrm{e}^{-\mathrm{i} G}=-\Delta-\mathrm{i}[-\Delta, G]+V+\text { l.h.t. }
$$


whose symbol has the form

$$
|\xi|^{2}+\left\{|\xi|^{2} ; g\right\}_{M}+V+\text { l.h.t. }
$$

where $\left\{|\xi|^{2} ; g\right\}_{M}$ is the so called Moyal bracket, which is the symbol of the operator $-\mathrm{i}[-\Delta, G]$. Furthermore, since $|\xi|^{2}$ is quadratic, the Moyal bracket coincides with the Poisson bracket, so the function $g$ constructed in (3.4) is suitable (after localization) in order to perform the semiclassical normal form of $H$.

Using $O p^{w}(g)$ in order to transform $H$ and iterating the procedure we conjugate $H$ to an operator with symbol

$$
|\xi|^{2}+z(\xi)+z^{(r e s)}(x, \xi)+O\left(|\xi|^{-N}\right),
$$

with some arbitrarily large $N$. Here $z^{(r e s)}$ is a symbol localized in the complement of $\Omega^{(0)}$.

As a last step, we use the equivalence of the Weyl quantization and the classical quantization in order to show that the operator obtained by quantizing (3.7) acts on $\mathrm{e}^{\mathrm{i} x \cdot \xi}$ with $\xi \in \mathbb{Z}^{d} \cap \Omega^{(0)}$ as a multiplication by $|\xi|^{2}+Z(\xi)$ plus an operator which is smoothing of order $N$. Thus $\mathrm{e}^{\mathrm{i} x \cdot \xi}$ is a quasimode for the quantization of (3.7) and Theorem 2 follows, at least in the case of Sturm-Liouville type operators. The case of a general torus is identical to the case just considered and the case where the main operator is $|\xi|^{M}$ is easily obtained by just remarking that the resonant zones of $|\xi|^{M}$ are the same as those of $|\xi|^{2}$.

\section{Some results on pseudo differential opera- tors}

First we give a few lemmas which are quite standard in the framework of pseudodifferential calculus (see e.g. [Rob87, Tay91] for pseudo-differential operators on $\mathbb{R}^{d}$ and [SV] for periodic pseudo-differential operators), and are here reformulated in a form suitable for our developments.

Lemma 4.1. Let $m, m^{\prime} \in \mathbb{R}, \delta>0, A=O p^{w}(a) \in O P S_{\delta}^{m}, B=O p^{w}(b) \in$

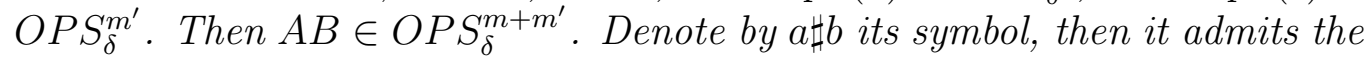
asymptotic expansion

$$
\begin{aligned}
& a \sharp b \sim \sum_{j \geq 0} \sigma_{j}(a, b), \\
& \sigma_{j}(a, b):=\frac{1}{\mathrm{i}^{j}} \sum_{|\alpha|+|\beta|=j}\left(\frac{1}{2}\right)^{|\alpha|}\left(-\frac{1}{2}\right)^{|\beta|}\left(\partial_{x}^{\beta} \partial_{\xi}^{\alpha} a\right)\left(\partial_{x}^{\alpha} \partial_{\xi}^{\beta} b\right) \in S_{\delta}^{m+m^{\prime}-\delta j}, \quad j \geq 0 .
\end{aligned}
$$


Furthermore one has

$$
\sigma_{j}(a, b)=(-1)^{j} \sigma_{j}(b, a) .
$$

If a is symmetric in $\xi$ and $b$ is skewsymmetric in $\xi$, then $\sigma_{j}(a, b)$ is symmetric for odd $j$ and skewsymmetric for even $j$.

Corollary 4.2. Denoting as usual by $\{a ; b\}_{M}:=\frac{1}{\mathrm{i}}(a \sharp b-b \sharp a)$ the symbol of $\frac{1}{\mathrm{i}}[A, B]$, one has

$$
\{a ; b\}_{M} \sim \sum_{j \geq 1} \sigma_{j}^{M}(a, b)
$$

with

$$
\sigma_{j}^{M}(a, b)=\left\{\begin{array}{cccc}
0 & \text { if } j & \text { even } \\
-2 \sigma_{j}(a, b) & \text { if } j & \text { odd }
\end{array} .\right.
$$

In particular one has

$$
\{a ; b\}_{M}=\{a ; b\}+S_{\delta}^{m+m^{\prime}-3 \delta}, \quad\{a ; b\}:=-\nabla_{\xi} a \cdot \nabla_{x} b+\nabla_{x} a \cdot \nabla_{\xi} b .
$$

Furthermore, if a is symmetric in $\xi$ and $b$ is skewsymmetric in $\xi$, then $\sigma_{j}^{M}$ is symmetric for any $j$.

Lemma 4.3 (Adjoint). Let $m \in \mathbb{R}, \delta>0, A=O p^{w}(a) \in O P S_{\delta}^{m}$. Then $A^{*}=O p^{w}(\bar{a}) \in O P S_{\delta}^{m}$. In particular if $a=\bar{a}$, the operator $O p^{w}(a)$ is self-adjoint. by

Given $\eta \in \mathbb{R}, \delta>0, g \in S_{\delta}^{\eta}$, we define the operator $a d_{g}^{M}: S_{\delta}^{m} \rightarrow S_{\delta}^{m+\eta-\delta}$

$$
a d_{g}^{M} a:=\{a ; g\}_{M} .
$$

We will also consider its powers $\left(a d_{g}^{M}\right)^{j}$, which are defined as usual. Remark that, for $a \in S_{\delta}^{m^{\prime}}$, according to Lemma 4.1, one has $\left(a d_{g}^{M}\right)^{j} a \in S_{\delta}^{m+j(\eta-\delta)}$. Remark that if $g$ is skewsymmetric in $\xi$ then $\left(a d_{g}^{M}\right)^{j}$ preserves the parity in $\xi$.

Given a selfadjoint pseudodifferential operator $G \in O P S_{\delta}^{\eta}$, we consider the unitary group generated by $-\mathrm{i} G$, which is denoted, as usual, by $\mathrm{e}^{-\mathrm{i} \tau G}$, $\tau \in \mathbb{R}$.

Definition 4.4. Given a unitary operator $\mathcal{U}$, we will say that it conjugates an operator $A$ to an operator $B$ if

$$
B=\mathcal{U} A \mathcal{U}^{-1}
$$

We recall the following simplified version of Egorov Theorem. 
Lemma 4.5. Fix $\eta \in \mathbb{R}$, and let $g \in S_{\delta}^{\eta}$, $0<\delta<1$ be a real valued symbol. Let $G=O p^{w}(g) \in O P S_{\delta}^{\eta}$. Then $\forall \tau \in[-1,1]$

(1) If $\eta<1$, then $e^{\mathrm{i} \tau G} \in \mathcal{B}\left(H^{s} ; H^{s}\right) \quad \forall s \geq 0$

(2) If $\eta<\delta, a \in S_{\delta}^{m}$ and $A=O p^{w}(a)$, then $H:=O p^{w}(h)=e^{\mathrm{i} \tau G} A e^{-\mathrm{i} \tau G} \in$ $O P S_{\delta}^{m}$ and its symbol admits the asymptotic expansion

$$
h \sim \sum_{j \geq 0} \frac{\tau^{j}\left(a d_{g}^{M}\right)^{j} a}{j !} .
$$

As a consequence the operator $H=O p^{w}(h):=e^{\mathrm{i} \tau G} A e^{-\mathrm{i} \tau G}$ admits the expansion

$$
h=a+\{a ; g\}+S_{\delta}^{m+2(\eta-\delta)} .
$$

(3) If a is even in $\xi$ and $g$ is skewsymmetric in $\xi$ then $h$ is even in $\xi$.

Finally we will need a lemma connecting the classical quantization and the Weyl quantization. We recall the following definition.

Definition 4.6 (Classical quantization). Given a symbol $a \in S_{\delta}^{m}$, we define its classical quantization $O p^{c l}(a)$ as follows: given $u \in C^{\infty}\left(\mathbb{T}_{\Gamma}^{d}\right)$, we put

$$
\left[O p^{c l}(a) u\right](x):=\sum_{\xi \in \Gamma^{*}} a(x, \xi) \widehat{u}(\xi) e^{\mathrm{i} x \cdot \xi}
$$

The following lemma is an $\hbar$ independent formulation of Theorem II-27 of [Rob87.

Lemma 4.7. Let $a \in S_{\delta}^{m}$. Then there exists a symbol $b \in S_{\delta}^{m}$ such that $O p^{w}(a)=O p^{c l}(b)$. Furthermore, the symbol $b$ admits the asymptotic expansion

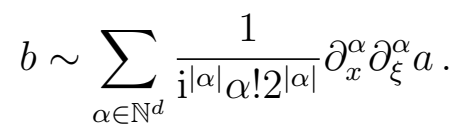

Remark 4.8. In particular, if a is a Fourier multiplier (i.e. independent of $x)$ one has that $O p^{w}(a)=O p^{c l}(a)$. Furthermore we have that, up to an operator in $S_{\delta}^{-\infty}, \operatorname{supp}(b) \subset \operatorname{supp}(a)$. 


\section{The normal form theorem and its proof}

To state the main result of this section we will use the constant $\rho$ defined by eq. (2.9), we fix $\gamma$ s.t.

$$
0<\gamma<r:=\frac{1}{2} \inf _{x \neq y \in \Gamma^{*}}|x-y|
$$

and define

$$
\Omega:=\left\{\xi \in \mathbb{R}^{d}:|\xi \cdot k|>\frac{2 \gamma\langle\xi\rangle^{\delta}}{|k|^{\tau}}, \quad \forall k \in \Gamma^{*}, \quad 0<|k|<\langle\xi\rangle^{\epsilon}\right\} .
$$

Remark that there exists a neighbourhood of the origin which does not intersect such a set.

Theorem 5.1. Let $\mathfrak{e}>0$ and let $\delta, \rho$ be two constants satisfying (2.8), 2.9. Then there exists a sequence of self-adjoint operators $\left(G_{n}\right)_{n \geq 1}$ with $G_{n}=$ $O p^{w}\left(g_{n}\right) \in O P S_{\delta}^{2-\mathfrak{e}-\delta-n \rho}$ for any $n \geq 1$, such that for any integer $n \geq 1$, the operator

$$
\mathcal{U}_{n}:=\mathrm{e}^{\mathrm{i} G_{n}} \circ \ldots \circ e^{\mathrm{i} G_{1}}
$$

conjugates $H$ to a pseudodifferential operator $H_{n}$ with symbol $h_{n}$ of the form

$$
h_{n}=h^{0}+z^{(n)}+v_{n},
$$

where $v_{n} \in S_{\delta}^{M-\mathfrak{e}-n \rho}$ and $z^{(n)} \in S_{\delta}^{M-\mathfrak{e}}$ is such that $z^{(n)}=\left\langle z^{(n)}\right\rangle+z^{(n, r e s)}$, with $\left\langle z^{(n)}\right\rangle$ independent of $x$ and $z^{(n, r e s)}(x, \xi)=0, \forall \xi \in \Omega$. Furthermore, for any integer $n \geq 0$, there exists a symbol $z_{n} \in S_{\delta}^{M-\mathfrak{e}-\rho n}$ such that

$$
\left\langle z^{(n)}\right\rangle(\xi)=\sum_{j=0}^{n-1} z_{j}(\xi) .
$$

Finally, if $h$ is symmetric in $\xi$, then the same is true for $v_{n}$ and $z_{n}$, whereas $g_{1}, \ldots, g_{n}$ are skew symmetric.

The rest of the section is split into few subsections and is devoted to the proof of this Theorem.

\subsection{Preliminaries and cutoffs}

We start by remarking that, given a symbol $a \in S_{\delta}^{m}$, the best constant $C_{\alpha . \beta}$ for which the inequality (2.4) holds is a seminorm of $a$. In case it is needed to make reference to the symbol $a$ we will write $C_{\alpha, \beta}(a)$. Furthermore, the space $S_{\delta}^{m}$ endowed by such a family of seminorms is a Fréchet space.

Sometimes we will write $a \lesssim b$ in order to mean there exists a constant $C$, independent of all the relevant quantities, s.t. $a \leq C b$. 
Remark 5.2. Let $a \in S_{\delta}^{m}$ then for any $\alpha \in \mathbb{N}^{d}, N \in \mathbb{N}$ one has that the Fourier coefficients $\widehat{a}(k, \xi)$ are estimated by

$$
\left|\partial_{\xi}^{\alpha} \widehat{a}(k, \xi)\right| \lesssim\langle k\rangle^{-N}\langle\xi\rangle^{m-\delta|\alpha|}, \quad \forall(k, \xi) \in \Gamma^{*} \times \mathbb{R}^{d} .
$$

Furthermore, defining $\hat{a}_{k}(x, \xi):=\widehat{a}(k, \xi) \mathrm{e}^{\mathrm{i} k \cdot x}$, one has a similar inequality, which implies that $\hat{a}_{k} \in S_{\delta}^{m}$ and, furthermore, for any $N$ one has

$$
C_{\alpha, \beta}\left(\hat{a}_{k}\right) \lesssim\langle k\rangle^{-N}
$$

We remark that the unwritten constant in the inequalities (5.6) and (5.7) depend on $N, m, \alpha, \beta, \delta$. Of course this dependence is irrelevant for our developments.

Remark 5.3. If $a \in S_{\delta}^{m}$ and $b \in S_{\delta}^{m^{\prime}}$, then $a b \in S_{\delta}^{m+m^{\prime}}$ and one has

$$
C_{\alpha, \beta}(a b) \lesssim\left[\sup _{\left|\alpha^{\prime}\right|+\left|\beta^{\prime}\right| \leq|\alpha|+|\beta|} C_{\alpha^{\prime}, \beta^{\prime}}(a)\right]\left[\sup _{\left|\alpha^{\prime}\right|+\left|\beta^{\prime}\right| \leq|\alpha|+|\beta|} C_{\alpha^{\prime}, \beta^{\prime}}(b)\right] .
$$

Let us consider an even cut-off function $\chi \in C_{c}^{\infty}(\mathbb{R})$ such that $\operatorname{supp}(\chi) \subseteq$ $[-2 \gamma, 2 \gamma], 0 \leq \chi \leq 1$ and $\chi(t)=1$ for any $t \in[-\gamma, \gamma]$. With its help we define, for any $k \in \Gamma^{*}$,

$$
\begin{gathered}
\chi_{k}(\xi):=\chi\left(\frac{2|k|^{\tau} \xi \cdot k}{\langle\xi\rangle^{\delta}}\right), \\
d_{k}(\xi):=\frac{1}{\xi \cdot k}\left(1-\chi_{k}(\xi)\right), \\
\tilde{\chi}_{k}(\xi):=\chi\left(\frac{|k|}{\langle\xi\rangle^{\varepsilon}}\right) .
\end{gathered}
$$

Lemma 5.4. The following estimates hold:

$$
\begin{aligned}
& \left|\chi_{k}(\xi)\right| \lesssim 1, \quad\left|\partial_{\xi}^{\beta} \chi_{k}(\xi)\right| \lesssim \frac{\langle k\rangle^{|\beta|(\tau+1)}}{\langle\xi\rangle^{|\beta| \delta}}, \quad \forall \beta \in \mathbb{N}^{d} \backslash\{0\}, \\
& \left|\partial_{\xi}^{\beta} d_{k}(\xi)\right| \lesssim \frac{\langle k\rangle^{(|\beta|+1) \tau+|\beta|}}{\langle\xi\rangle^{\delta(|\beta|+1)}}, \quad \forall \beta \in \mathbb{N}^{d}, \\
& \left|\tilde{\chi}_{k}(\xi)\right| \lesssim 1, \quad\left|\partial_{\xi}^{\beta} \tilde{\chi}_{k}(\xi)\right| \lesssim \frac{\langle k\rangle^{|\beta|}}{\langle\xi\rangle^{\varepsilon+|\beta|}}, \quad \forall \beta \in \mathbb{N}^{d} \backslash\{0\},
\end{aligned}
$$

Remark 5.5. The above lemma implies that

$\chi_{k} \in S_{\delta}^{0}, \quad$ with seminorms $C_{\alpha, \beta}\left(\chi_{k}\right) \lesssim\langle k\rangle^{|\beta|(\tau+1)}$, $d_{k} \in S_{\delta}^{-\delta}$, with seminorms $C_{\alpha, \beta}\left(d_{k}\right) \lesssim\langle k\rangle^{(|\beta|+1) \tau+|\beta|}$,

$\tilde{\chi}_{k} \in S_{\delta}^{0}, \quad$ with seminorms $C_{\alpha, \beta}\left(\tilde{\chi}_{k}\right) \lesssim\langle k\rangle^{|\beta|}$, 
Proof. Estimates of $\chi_{k}$. Clearly, by the definition of the cut-off function $\chi$, one has that $\chi_{k}$ is uniformly bounded by 1 . Let $\beta \in \mathbb{N}^{d} \backslash\{0\}$ be a multiindex of lenght 1 . Then

$$
\begin{aligned}
\left|\partial_{\xi}^{\beta} \chi_{k}(\xi)\right| & \lesssim\left|\chi^{\prime}\left(\frac{|k|^{\tau} \xi \cdot k}{\langle\xi\rangle^{\delta}}\right)\langle k\rangle^{\tau}\right|\left|\partial_{\xi}^{\beta}\left(\frac{\xi \cdot k}{\langle\xi\rangle^{\delta}}\right)\right| \\
& \lesssim \frac{\langle k\rangle^{\tau+1}}{\langle\xi\rangle^{\delta}}
\end{aligned}
$$

The estimates for the higher order derivatives is analogous.

Estimates of $d_{k}$ Note that by the definition (5.9), one has that

$$
\operatorname{supp}\left(d_{k}\right) \subseteq\left\{(k, \xi) \in \Gamma^{*} \times \mathbb{R}^{d}:|\xi \cdot k| \geq \frac{\langle\xi\rangle^{\delta}}{\langle k\rangle^{\tau}}\right\}
$$

This implies that

$$
\left|d_{k}(\xi)\right| \lesssim \frac{\langle k\rangle^{\tau}}{\langle\xi\rangle^{\delta}}
$$

Furthermore, for any multiindex $\beta \in \mathbb{N}^{d} \backslash\{0\}$ of lenght one, one has that

$$
\left|\partial_{\xi}^{\beta} d_{k}(\xi)\right| \lesssim \frac{\langle k\rangle}{|\xi \cdot k|^{2}}\left|1-\chi_{k}(\xi)\right|+\frac{1}{|\xi \cdot k|}\left|\partial_{\xi}^{\beta} \chi_{k}(\xi)\right| .
$$

By (5.13), one has that $\frac{1}{|\xi \cdot k|} \lesssim \frac{\langle k\rangle^{\tau}}{\langle\xi\rangle^{\delta}}$, for any $(k, \xi) \in \operatorname{supp}\left(d_{k}\right)$, therefore, using also that $\left|\partial_{\xi}^{\beta} \chi_{k}(\xi)\right| \lesssim\langle k\rangle^{\tau+1}\langle\xi\rangle^{-\delta}$, one obtains

$$
\left|\partial_{\xi}^{\beta} d_{k}(\xi)\right| \lesssim \frac{\langle k\rangle^{2 \tau+1}}{\langle\xi\rangle^{2 \delta}}
$$

The estimate of $\tilde{\chi}_{k}$ is similar to the others and is omitted.

Given $m \in \mathbb{R}, \delta>0, a \in S_{\delta}^{m}$, we define

$$
\begin{aligned}
& \langle a\rangle(\xi):=\frac{1}{\left|\mathbb{T}_{\Gamma}^{d}\right|} \int_{\mathbb{T}_{\Gamma}^{d}} a(x, \xi) d x, \\
& a^{(r e s)}(x, \xi):=\sum_{k \in \Gamma^{*} \backslash\{0\}} \chi_{k}(\xi) \tilde{\chi}_{k}(\xi) \widehat{a}(k, \xi) e^{\mathrm{i} k \cdot x}, \\
& a^{(n r)}(x, \xi):=\sum_{k \in \Gamma^{*} \backslash\{0\}}\left(1-\chi_{k}(\xi)\right) \tilde{\chi}_{k}(\xi) \widehat{a}(k, \xi) e^{\mathrm{i} k \cdot x}, \\
& a^{(S)}(x, \xi):=\sum_{k \in \Gamma^{*} \backslash\{0\}}\left(1-\tilde{\chi}_{k}(\xi)\right) \widehat{a}(k, \xi) e^{\mathrm{i} k \cdot x},
\end{aligned}
$$

so that one has

$$
a=\langle a\rangle+a^{(n r)}+a^{(r e s)}+a^{(S)} .
$$


Lemma 5.6. One has that $\langle a\rangle, a^{(r e s)}, a^{(n r)} \in S_{\delta}^{m}$ and $a^{(S)} \in S^{-\infty}$.

Proof. We prove the statement for $a^{(r e s)}$, all the other estimates are obtained in the same way.

By the definition of $a^{(r e s)}$ and recalling the notation introduced in Remark (5.2), one has $a^{(r e s)}=\sum_{k} \chi_{k} \tilde{\chi}_{k} \hat{a}_{k}$. The general term of the series is in $S_{\delta}^{m}$ and, by the estimate (5.7) and Lemma 5.5, its seminorms are estimated by

$$
C_{\alpha, \beta}\left(\chi_{k} \tilde{\chi}_{k} \hat{a}_{k}\right) \lesssim \frac{\langle k\rangle^{(|\beta|+1) \tau+|\beta|}\langle k\rangle^{|\beta|}}{\langle k\rangle^{N}} \lesssim \frac{1}{\langle k\rangle^{N^{\prime}}},
$$

with an arbitrary $N^{\prime}$. Therefore, the series converges in any seminorm and therefore $a^{(r e s)} \in S_{\delta}^{m}$. Furthermore, its Fourier coefficients still fulfill (5.6).

We come to the normal form procedure. First, in order to regularize the singularity at the origin of the derivatives, we substitute $|\xi|^{M}$ with

$$
h^{0}(\xi):=\psi(\xi)|\xi|^{M}
$$

where $\psi \in C^{\infty}(\mathbb{R})$ is an even cut-off function such that $\psi(t)=0$. for any $t \in[-\gamma, \gamma], 0 \leq \psi \leq 1$ and $\psi(t)=1$ for any $|t| \geq 2 \gamma$, where $\gamma$ is the constant given in (5.1). Note that by using such a definition of $h^{0}$, for any function $u \in L^{2}\left(\mathbb{T}_{\Gamma}^{d}\right)$, one has that

$$
O p^{w}\left(h^{0}\right) u(x)=\sum_{\xi \in \Gamma^{*}}|\xi|^{M} \widehat{u}(\xi) e^{\mathrm{i} x \cdot \xi} .
$$

Remark 5.7. For any $\alpha \in \mathbb{N}^{d}$, a direct calculation shows that

$$
\left|\partial_{\xi}^{\alpha} h^{0}(\xi)\right| \lesssim|\xi|^{M-|\alpha|}, \quad \forall \xi \in \mathbb{R}^{d} .
$$

\subsection{The normal form construction.}

Then, given $a \in S_{\delta}^{m}$, consider

$$
g(x, \xi):=\sum_{k \in \Gamma^{*} \backslash\{0\}} \psi(\xi) \tilde{\chi}_{k}(\xi)|\xi|^{2-M}(-\mathrm{i}) d_{k}(\xi) \widehat{a}(k, \xi) e^{\mathrm{i} k \cdot x}
$$

where we recall the definitions given in $(5.9)$.

The following Lemma is easily seen to hold

Lemma 5.8. The symbol $g$ defined in (5.19) belongs to class $S_{\delta}^{2+m-M-\delta}$ and it satisfies

$$
\left\{h^{0} ; g\right\}+a^{(n r)} \in S_{\delta}^{-\infty} .
$$

Moreover, if a is symmetric in $\xi$, then, since $d_{k}(\xi)$ is skew symmetric, $g$ is skew symmetric too. 
Proof. Recall the definition of $h^{0}$ in (5.17). Using that the cut-off function $\psi$ is a smooth function with compact support, one has that $\nabla_{\xi}\left(\psi(\xi)|\xi|^{M}\right)=$ $M|\xi|^{M-2} \xi \psi(\xi)+S^{-\infty}$, therefore

$$
\left\{h^{0} ; g\right\}(x, \xi)=-|\xi|^{M-2} \psi(\xi) \xi \cdot \nabla_{x} g(x, \xi)+S_{\delta}^{-\infty} .
$$

In order to solve the equation (5.20), it is enough to solve

$$
-|\xi|^{M-2} \psi(\xi) \xi \cdot \nabla_{x} g+a^{(n r)} \in S_{\delta}^{-\infty} .
$$

Recalling the definition of $a^{(n r)}$ given in $(5.15)$ and the definitions given in $(5.9)$, a solution of the equation $(5.22)$ is then given by $g$ defined in (5.19). By Lemma 5.5, one gets that the general term in the sum at r.h. in equation $(5.19)$ belongs to $S_{\delta}^{2+m-M-\delta}$ and for any $\alpha, \beta \in \mathbb{N}$ and $N \in \mathbb{N}$ large enough (depending on $\alpha, \beta$ ) its seminorms decay as $\langle k\rangle^{-N}$, implying that $g \in S_{\delta}^{2+m-M-\delta}$. Finally, if $a$ is even in $\xi$, using that $\tilde{\chi}_{k}$ and $\psi$ are even and $d_{k}$ is odd, one gets that $g$ is skew symmetric in $\xi$ and the proof is concluded.

Proof of Theorem 5.1. We describe the induction step of the normal form procedure which allows to Prove Theorem 5.1. Assume that $H_{n}=O p^{w}\left(h_{n}\right)$ has the form given in (5.4) with $z_{n}=\left\langle z_{n}\right\rangle+z_{n}^{(\text {res })} \in S_{\delta}^{M-\mathfrak{e}}$ and $v_{n} \in S_{\delta}^{M-\mathfrak{e}-n \rho}$. By Lemma 5.8 there exists a solution

$$
g_{n+1} \in S_{\delta}^{2-\mathfrak{e}-\delta-n \rho}
$$

of the homological equation

$$
\left\{h^{0} ; g_{n+1}\right\}+v_{n}^{(n r)} \in S_{\delta}^{-\infty} .
$$

Moreover since the symbol $v_{n}$ is real valued, then also the symbol $v_{n}^{(n r)}$ is real valued and therefore $g_{n+1}=\bar{g}_{n+1}$. Then, we define $G_{n+1}:=O p^{w}\left(g_{n+1}\right)$. Note that $G_{n+1}$ is self-adjoint since the symbol $g_{n+1}$ is real valued. Since, by (2.8), $\delta>1-\frac{\mathfrak{e}}{4}>1-\frac{\mathfrak{e}}{2}$, one obtains that $2-\mathfrak{e}-\delta-n \rho<\delta<1$. Hence by Lemma 4.5, $e^{\mathrm{i} G_{n}}, e^{-\mathrm{i} G_{n}}$ are well defined linear operators in $\mathcal{B}\left(H^{s}\right)$ for any $s \geq 0$ and $H_{n+1}=O p^{w}\left(h_{n+1}\right)=e^{\mathrm{i} G_{n+1}} H_{n} e^{-\mathrm{i} G_{n+1}} \in O P S_{\delta}^{M}$. Furthermore, by applying (4.4) (with $a=h_{n}, g=g_{n+1}, m=M, \eta=2-\mathfrak{e}-\delta-n \rho$ ), one gets that $h_{n+1}$ admits the expansion

$$
h_{n+1}=h_{n}+\left\{h_{n} ; g_{n+1}\right\}+S_{\delta}^{M+4-2 \mathfrak{e}-4 \delta-2 n \rho} .
$$

Using that, by $(2.9)$, one gets that $M+4-2 \mathfrak{e}-4 \delta-2 n \rho \leq M-\mathfrak{e}-(n+1) \rho$, implying that $S_{\delta}^{M+4-2 \mathfrak{e}-4 \delta-2 n \rho} \subseteq S_{\delta}^{M-\mathfrak{e}-(n+1) \rho}$, hence

$$
h_{n+1}=h_{n}+\left\{h_{n} ; g_{n+1}\right\}+S_{\delta}^{M-\mathfrak{e}-(n+1) \rho} .
$$


Moreover, by (5.4), using the splitting (5.15) and Lemma 5.6, one has

$$
\begin{aligned}
h_{n}+\left\{h_{n} ; g_{n+1}\right\}= & h^{0}+z_{n}+\left\langle v_{n}\right\rangle+v_{n}^{(r e s)}+v_{n}^{(n r)}+\left\{h^{0} ; g_{n+1}\right\} \\
& +\left\{z_{n} ; g_{n+1}\right\}+\left\{v_{n} ; g_{n+1}\right\}+S_{\delta}^{-\infty} .
\end{aligned}
$$

By 5.23 and using that $z_{n} \in S_{\delta}^{M-\mathfrak{e}}, v_{n} \in S_{\delta}^{M-\mathfrak{e}-n \rho} \subseteq S_{\delta}^{M-\mathfrak{e}}$, one gets

$$
v_{n}^{(n r)}+\left\{h^{0} ; g_{n+1}\right\} \in S_{\delta}^{-\infty}, \quad\left\{z_{n}+v_{n} ; g_{n+1}\right\} \in S_{\delta}^{M-\mathfrak{e}-n \rho-(2 \delta+\mathfrak{e}-2)} .
$$

Note that since $\delta<1$, one has that $2 \delta+\mathfrak{e}-2>4 \delta+\mathfrak{e}-4=\rho$ and therefore $\left\{g_{n+1} ; z_{n}+v_{n}\right\} \in S_{\delta}^{M-\mathfrak{e}-(n+1) \rho}$. Hence (5.25), (5.26) imply that

$$
h_{n+1}=h^{0}+z_{n+1}+S^{M-\mathfrak{e}-(n+1) \rho}
$$

where $z_{n+1}=\left\langle z_{n+1}\right\rangle+z_{n+1}^{(\text {res })}$, with $\left\langle z_{n+1}\right\rangle:=\left\langle z_{n}\right\rangle+\left\langle v_{n}\right\rangle$ and $z_{n+1}^{(\text {res })}:=z_{n}^{(\text {res })}+$ $v_{n}^{(\text {res })}$. The expansions (5.4), (5.5) are then proved at the step $n+1$. Finally, if $v_{n}, z_{n}$ are symmetric in $\xi$, then Lemma 5.8 implies that $g_{n+1}$ is skew symmetric in $\xi$, hence, by applying Lemma 4.5, $z_{n+1}, v_{n+1}$ are symmetric in $\xi$.

\subsection{Measure estimates of the non resonant set}

In this section we prove that the non resonant set $\Omega$ introduced in Theorem 2.4 is of density one. Recall that, according to Definition (5.2),

$$
\Omega:=\left\{\xi \in \mathbb{R}^{d}:|\xi \cdot k|>\frac{2 \gamma\langle\xi\rangle^{\delta}}{|k|^{\tau}}, \quad \forall k \in \Gamma^{*}, \quad 0<|k|<\langle\xi\rangle^{\epsilon}\right\} .
$$

In particular, we prove the following

Proposition 5.9. Assume $\epsilon \leq \frac{\delta}{1+\tau}, \tau>d-1$ and $R>\left[r 2^{\epsilon(\tau+1)}\right]^{1 /(\delta-\epsilon(\tau+1))}$. Then one has

$$
1-\frac{\sharp\left(\Omega \cap \Gamma^{*} \cap B_{R}(0)\right)}{\sharp\left(\Gamma^{*} \cap B_{R}(0)\right)}=\mathcal{O}\left(R^{\delta-1}\right) \text {. }
$$

Given a (measurable) set $\mathcal{A}$, and a positive parameter $r$ we will denote

$$
\mathcal{A}_{r}:=\bigcup_{x \in \mathcal{A}} B_{r}(x)
$$

We start by a few remarks that will be useful in order to estimate the cardinality of $\Omega \cap \Gamma^{*}$. 
Remark 5.10. There exists a constant $C$ s.t.

$$
\sharp\left(\Gamma^{*} \cap B_{R}\right) \geq C R^{d} .
$$

Remark 5.11. Let $E=\left\{x_{1}, \ldots, x_{N}\right\} \subset \mathbb{R}^{d}$, be a finite subset and for $r:=$ $\inf _{i \neq j} \frac{\left|x_{i}-x_{j}\right|}{2}$, consider the set $E_{r}$ (defined according to (5.28)). Then

$$
N \equiv \sharp E=\frac{\left|E_{r}\right|}{\left|B_{r}(0)\right|}=\frac{\left|E_{r}\right|}{\left|B_{1}(0)\right| r^{d}} .
$$

Recall the definition of $r$ as in (5.1); clearly, one has that for any $\xi_{0} \in \Gamma^{*}$, $B_{r}\left(\xi_{0}\right) \cap\left(\Gamma^{*} \backslash\left\{\xi_{0}\right\}\right)=\emptyset$.

Remark 5.12. Given a measurable set, $\mathcal{A}$, we have

$$
\sharp\left(\mathcal{A} \cap \Gamma^{*}\right) \leq \frac{\left|\mathcal{A}_{r}\right|}{\left|B_{r}(0)\right|} .
$$

Remark 5.13. By the above remark one also has

$$
\sharp\left(\Gamma^{*} \cap B_{R}(0)\right) \lesssim R^{d} .
$$

Let

$$
\Omega^{(R)}:=\Omega \cap B_{R} \quad \text { and } \quad \Omega^{(R, c)}:=B_{R} \backslash \Omega^{(R)} ;
$$

In order to estimate the cardinality of $\Omega^{(R, c)} \cap \Gamma^{*}$ we estimate the measure of $\Omega_{r}^{(R, c)}$. To this end we remark that

$$
\Omega_{r}^{(R, c)} \subset \bigcup_{0<|k|<(R+r)^{\epsilon}} A_{k, r}, \quad A_{k}:=\left\{\xi \in B_{R}:|\xi \cdot k|<\frac{2 \gamma R^{\delta}}{|k|^{\tau}}\right\}_{r},
$$

and $A_{k, r}$ is the extension of $A_{k}$ according to (5.28). In order to estimate the measure of $A_{k, r}$ we will use the following Lemma.

Lemma 5.14. Assume $\epsilon \leq \frac{\delta}{1+\tau}$ and $R>\left[r 2^{\epsilon(\tau+1)} / 2 \gamma\right]^{1 /(\delta-\epsilon(\tau+1))}$. Then for any $k \in \Gamma^{*}, 0<|k|<(2 R)^{\epsilon}$, one has that

$$
A_{k, r} \subset \widetilde{A}_{k}:=\left\{\xi \in B_{2 R}:|\xi \cdot k|<\frac{4 \gamma R^{\delta}}{|k|^{\tau}}\right\} .
$$

Proof. Let $\xi^{\prime} \in A_{k, r}$, then there exist $\xi \in A_{k}$ and $h \in B_{r}(0)$ s.t. $\xi^{\prime}=\xi+h$. First one has $\xi^{\prime} \in B_{2 R}(0)$ provided $r<R$. Furthermore one has

$$
|(\xi+h) \cdot k| \leq|\xi \cdot k|+|h \cdot k| \stackrel{\xi \in A_{k}}{<} \frac{2 \gamma R^{\delta}}{|k|^{\tau}}+|h||k| \leq \frac{2 \gamma R^{\delta}}{|k|^{\tau}}+r(2 R)^{\epsilon} .
$$

One has that

$$
\frac{2 \gamma R^{\delta}}{|k|^{\tau}}+r(2 R)^{\epsilon} \leq \frac{4 \gamma R^{\delta}}{|k|^{\tau}}
$$

by taking $r 2^{\epsilon(\tau+1)} / 2 \gamma<R^{\delta-\epsilon(1+\tau)}$ which is implied by the assumption. 
Proposition 5.15. Assume $\epsilon \leq \frac{\delta}{1+\tau}, \tau>d-1$ and $R>\left[r 2^{\epsilon(\tau+1)} / 2 \gamma\right]^{1 /(\delta-\epsilon(\tau+1))}$. Then one has

$$
\left|\Omega_{r}^{(R, c)}\right| \lesssim R^{d+\delta-1}
$$

Proof. The proof is standard, we give it here for the sake of completeness. Since $\widetilde{A}_{k}$ as defined in (5.33) is the intersection of a layer of thickness $4 R^{\delta} /|k|^{\tau}|k|$ with a sphere of radius $R$, we have

$$
\left|\widetilde{A}_{k}\right| \lesssim \frac{R^{\delta}}{|k|^{\tau+1}} R^{d-1}
$$

thus, having fixed some large $R_{1}$, we have

$$
\begin{aligned}
& \quad\left|\bigcup_{|k| \leq(2 R)^{\epsilon}} \widetilde{A}_{k}\right| \leq\left|\bigcup_{|k| \in \Gamma^{*} \backslash\{0\}} \widetilde{A}_{k}\right| \leq \sum_{|k| \in \Gamma^{*} \backslash\{0\}}\left|\widetilde{A}_{k}\right| \\
& \lesssim \sum_{|k| \in \Gamma^{*} \backslash\{0\}} \frac{R^{\delta}}{|k|^{\tau+1}} R^{d-1} \lesssim R^{\delta+d-1} \sum_{l=0}^{+\infty} \sum_{l R_{1}<|k| \leq(l+1) R_{1}} \frac{1}{|k|^{\tau}} \\
& \lesssim R^{\delta+d-1}\left(\sum_{0<|k| \leq R_{1}} \frac{1}{|k|^{\tau}}+\sum_{l=1}^{+\infty} \sum_{l R_{1}<|k| \leq(l+1) R_{1}} \frac{1}{\left(l R_{1}\right)^{\tau}}\right) .
\end{aligned}
$$

Exploiting again Remark 5.12 , we get, for $l \geq 1$,

$$
\sharp\left[\left(B_{(l+1) R_{1}} \backslash B_{l R_{1}}\right) \cap \Gamma^{*}\right] \lesssim\left|B_{(l+1) R_{1}} \backslash B_{l R_{1}}\right| \lesssim l^{d-1} R_{1}^{d},
$$

and thus the bracket at r.h.s. of (5.36) is bounded by a constant and the proposition holds.

We finally show the following

Proposition 5.16. For any $R>\left[r 2^{\epsilon(\tau+1)}\right]^{1 /(\delta-\epsilon(\tau+1))}$, one has that

$$
\frac{\sharp\left(\Omega \cap B_{R} \cap \Gamma^{*}\right)}{\sharp\left(B_{R} \cap \Gamma^{*}\right)}=1-O\left(R^{\delta-1}\right) .
$$

As a consequence, since $0<\delta<1$,

$$
\lim _{R \rightarrow+\infty} \frac{\sharp\left(\Omega \cap B_{R} \cap \Gamma^{*}\right)}{\sharp\left(B_{R} \cap \Gamma^{*}\right)}=1 .
$$


Proof. By recalling the formula (5.31), one has that

$$
\sharp\left(\Omega \cap B_{R} \cap \Gamma^{*}\right)=\sharp\left(\Omega^{(R)} \cap \Gamma^{*}\right)=\sharp\left(B_{R} \cap \Gamma^{*}\right)-\sharp\left(\Omega^{(R, c)} \cap \Gamma^{*}\right) .
$$

By Remark 5.12 and Lemma 5.15 , one obtains that

$$
\sharp\left(\Omega^{(R, c)} \cap \Gamma^{*}\right) \lesssim R^{d+\delta-1}
$$

and therefore, using Remark 5.30 and the formula (5.38) one obtains the claimed estimate (5.37).

\section{Proof of Theorem 2.4}

The estimate 2.11 follows by Proposition 5.16.

We show now that for $\xi \in \Omega, e^{\mathrm{i} k \cdot \xi}$ is a quasimode for $H_{n}$. By the normal form Theorem 5.1, for any $n \in \mathbb{N}$, there exists a unitary map $\mathcal{U}_{n} \in \mathcal{B}\left(H^{s}\right)$ for any $s \geq 0$ such that the $H_{n}=O p^{w}\left(h_{n}\right)=\mathcal{U}_{n} H_{0} \mathcal{U}_{n}^{-1}$ satisfies the expansion given in (5.4), namely

$$
h_{n}=h^{0}+\left\langle z_{n}\right\rangle+z_{n}^{(r e s)}+v_{n}
$$

with $\left\langle z_{n}\right\rangle, z_{n}^{(\text {res })} \in S_{\delta}^{M-\mathfrak{e}}, v_{n} \in S_{\delta}^{M-\mathfrak{e}-\rho n}$ and $\operatorname{supp}\left(z_{n}^{(r e s)}\right) \cap \Omega=\emptyset$ according to Theorem 5.1. By applying Lemma 4.7, one has that there exists $\widetilde{z}_{n}^{(r e s)} \in$ $S_{\delta}^{M-\mathfrak{e}}, \widetilde{v}_{n} \in S_{\delta}^{M-\mathfrak{e}-\rho n}$ such that

$$
\begin{aligned}
& O p^{w}\left(z_{n}^{(r e s)}\right)=O p^{c l}\left(\widetilde{z}_{n}^{(r e s)}\right), \quad O p^{w}\left(v_{n}\right)=O p^{c l}\left(\widetilde{v}_{n}\right), \\
& \operatorname{supp}\left(\widetilde{z}_{n}^{(r e s)}\right)=\operatorname{supp}\left(z_{n}^{(r e s)}\right) \subseteq \mathbb{R}^{d} \backslash \Omega, \quad \operatorname{supp}\left(v_{n}\right)=\operatorname{supp}\left(\widetilde{v}_{n}\right) .
\end{aligned}
$$

Therefore, given $\xi \in \Omega \cap \Gamma^{*}$, one gets, by explicit computation exploiting the definition of $O p^{w}$,

$$
O p^{w}\left(z_{n}^{(r e s)}\right)\left[e^{\mathrm{i} x \cdot \xi}\right]=O p^{c l}\left(\widetilde{z}_{n}^{(r e s)}\right)\left[e^{\mathrm{i} x \cdot \xi}\right]=0
$$

since $\widetilde{z}_{n}^{(r e s)}(x, \xi)=0$ for any $\xi \in \Omega$. Moreover, one has that

$$
O p^{w}\left(v_{n}\right)\left[e^{\mathrm{i} x \cdot \xi}\right]=O p^{c l}\left(\widetilde{v}_{n}^{(r e s)}\right)\left[e^{\mathrm{i} x \cdot \xi}\right]=\widetilde{v}_{n}^{(r e s)}(x, \xi) e^{\mathrm{i} x \cdot \xi}=O\left(\langle\xi\rangle^{M-\mathfrak{e}-\rho n}\right) .
$$

Hence 6.1 -6.3 imply that for any $\xi \in \Omega \cap \Gamma^{*}$

$$
H_{n}\left[e^{\mathrm{i} x \cdot \xi}\right]=\lambda_{n}(\xi) e^{\mathrm{i} x \cdot \xi}+O\left(\langle\xi\rangle^{M-\mathfrak{e}-\rho n}\right), \quad \lambda_{n}(\xi):=h^{0}(\xi)+\left\langle z_{n}\right\rangle(\xi)
$$


The existence of one eigenvalue $O\left(\langle\xi\rangle^{M-\mathfrak{e}-\rho n}\right)$ close to $\lambda_{n}(\xi)$ follows by the standard quasimode argument. In the case with symmetry, we need to construct two eigenvalues bifurcating from $\lambda_{n}(\xi)$ (note that $\lambda_{n}(\xi)$ is even in $\xi)$. This situation was studied in [BKP15]. According to Proposition 5.1, statement (ii) of that paper, the result follows from the fact that in such a case $\left\langle e^{\mathrm{i} \xi \cdot x}, e^{\mathrm{i} \xi^{\prime} \cdot x}\right\rangle_{L^{2}}=0$. Of course the same is true in the case of higher multiplicity.

We also remark that, defining $\varphi_{n, \xi}:=\mathcal{U}_{n}^{-1}\left[e^{\mathrm{i} x} \cdot \xi\right]$, it is a quasimode for the original Hamiltonian. Indeed one has

$$
H_{0} \varphi_{n, \xi}=\mathcal{U}_{n}^{-1} H_{n} \mathcal{U}_{n}\left[\varphi_{n, \xi}\right]=\lambda_{n}(\xi) \varphi_{n, \xi}+O\left(\langle\xi\rangle^{M-\mathfrak{e}-\rho n}\right)
$$

\section{References}

[Bam04] D. Bambusi. Semiclassical normal forms. In Multiscale methods in quantum mechanics, Trends Math., pages 23-39. Birkhäuser Boston, Boston, MA, 2004.

[Bam17] D. Bambusi. Reducibility of 1-d Schrödinger equation with time quasiperiodic unbounded perturbations, II. Comm. Math. Phys., 353(1):353-378, 2017.

[Bam18] D. Bambusi. Reducibility of 1-d Schrödinger equation with time quasiperiodic unbounded perturbations, I. Trans. Amer. Math. Soc., 370(3):1823-1865, 2018.

[BBM14] P. Baldi, M. Berti, and R. Montalto. KAM for quasi-linear and fully nonlinear forced perturbations of Airy equation. Math. Ann., 359(1-2):471-536, 2014.

[BGMR18] D. Bambusi, B. Grebert, A. Maspero, and D. Robert. Reducibility of the quantum Harmonic oscillator in $d$-dimensions with polynomial time dependent perturbation. Analysis \& PDEs, 11(3):775-799, 2018.

[BKP15] D. Bambusi, T. Kappeler, and T. Paul. From Toda to KdV. Nonlinearity, 28(7):2461-2496, 2015.

[BM18] Dario Bambusi and Riccardo Montalto. Reducibility of 1-d Schrödinger equation with time quasiperiodic unbounded perturbations, III. J. Math. Phys., 59(12):122702., 2018. 
[FKT90] Joel Feldman, Horst Knörrer, and Eugene Trubowitz. The perturbatively stable spectrum of a periodic Schrödinger operator. Invent. Math., 100(2):259-300, 1990.

[Fri90] Leonid Friedlander. On the spectrum of the periodic problem for the Schrödinger operator. Comm. Partial Differential Equations, 15(11):1631-1647, 1990.

[Kar96] Yu. E. Karpeshina. Perturbation series for the Schrödinger operator with a periodic potential near planes of diffraction. Comm. Anal. Geom., 4(3):339-413, 1996.

[Kar97] Yulia E. Karpeshina. Perturbation theory for the Schrödinger operator with a periodic potential, volume 1663 of Lecture Notes in Mathematics. Springer-Verlag, Berlin, 1997.

[PS10] Leonid Parnovski and Alexander V. Sobolev. Bethe-Sommerfeld conjecture for periodic operators with strong perturbations. Invent. Math., 181(3):467-540, 2010.

[Rob87] D. Robert. Autour de l'approximation semi-classique. PM 68. Birkhäuser, 1987.

[Roy07] Nicolas Roy. A semi-classical K. A. M. theorem. Comm. Partial Differential Equations, 32(4-6):745-770, 2007.

[Tay91] Michael E. Taylor. Pseudodifferential operators and nonlinear PDE, volume 100 of Progress in Mathematics. Birkhäuser Boston, Inc., Boston, MA, 1991.

[SV] J. Saranen, G. Vainikko. Periodic Integral and Pseudodifferential Equations with Numerical Approximation. Springer Monographs in Mathematics, 2002.

[Vel15] Oktay Veliev. Multidimensional periodic Schrödinger operator, volume 263 of Springer Tracts in Modern Physics. Springer, Cham, 2015. Perturbation theory and applications.

[Wan11] W.-M. Wang. Eigenfunction localization for the 2D periodic Schrödinger operator. Int. Math. Res. Not. IMRN, (8):18041838, 2011.

[W] A. Weinstein. Asymptotics of eigenvalue clusters for the Laplacian plus a potential. Duke Math. J. 44 (1977), no. 4, 883-892. 